US Army Corps

of Engineers ${ }_{\circledast}$

Engineer Research and

Development Center

\title{
Phase-Field Modeling of Nonequilibrium Solidification Processes in Additive Manufacturing
}

Jeffrey B. Allen, Robert D. Moser, Zackery B. McClelland, Md Mohaiminul Islam, and Ling Liu

November 2021

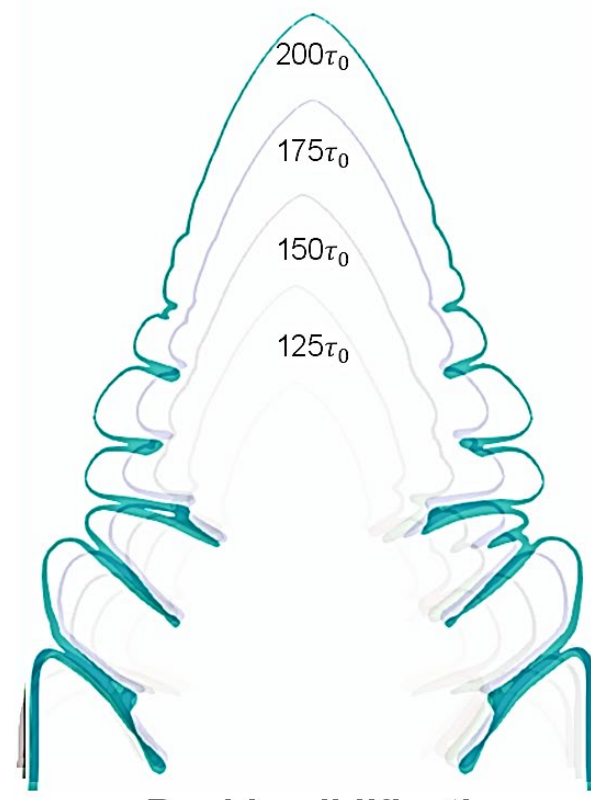

Rapid solidification

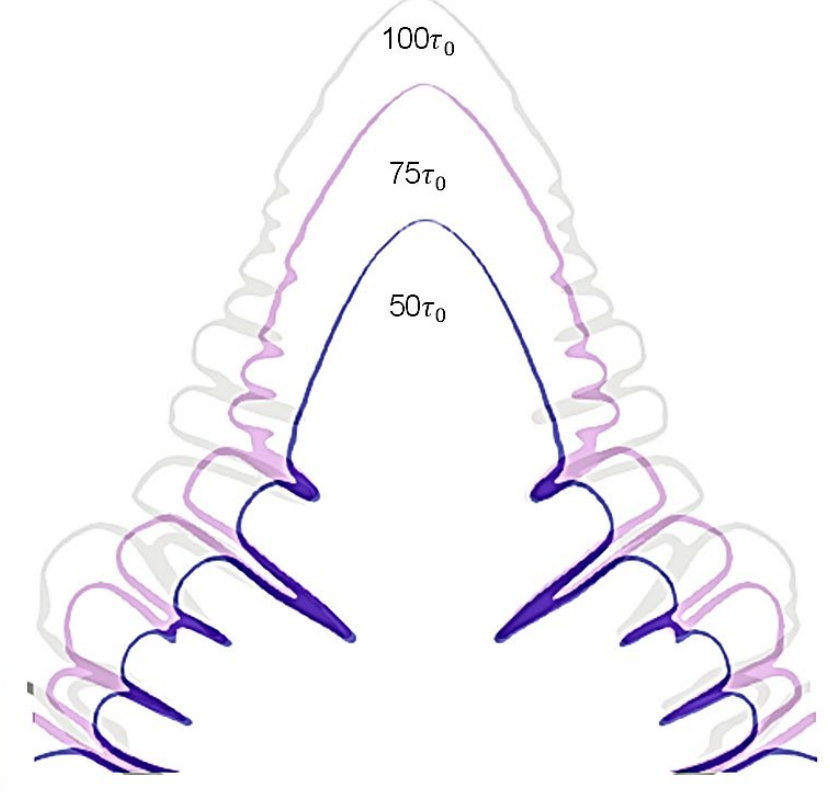

Slow solidification 
The U.S. Army Engineer Research and Development Center (ERDC) solves the nation's toughest engineering and environmental challenges. ERDC develops innovative solutions in civil and military engineering, geospatial sciences, water resources, and environmental sciences for the Army, the Department of Defense, civilian agencies, and our nation's public good. Find out more at www.erdc.usace.army.mil.

To search for other technical reports published by ERDC, visit the ERDC online library at https://erdclibrary.on.worldcat.org/discovery. 


\section{Phase-Field Modeling of Nonequilibrium Solidification Processes in Additive Manufacturing}

Jeffrey B. Allen

Information Technology Laboratory

U.S. Army Engineer Research and Development Center 3909 Halls Ferry Road

Vicksburg, MS 39180-6199

Robert D. Moser and Zackery B. McClelland

Geotechnical and Structures Laboratory

U.S. Army Engineer Research and Development Center 3909 Halls Ferry Road

Vicksburg, MS 39180-6199

Md Mohaiminul Islam and Ling Liu

Mechanical Engineering

Temple University

1947 N 12th St

Philadelphia, PA 19122 USA

Final Report

Approved for public release; distribution is unlimited.

Prepared for Information Technology Laboratory U.S. Army Engineer Research and Development Center 3909 Halls Ferry Road, Vicksburg, MS 39180-6199

Under PE 9C2284/Project 490803/Task A1020 


\section{Abstract}

This project models dendrite growth during nonequilibrium solidification of binary alloys using the phase-field method (PFM). Understanding the dendrite formation processes is important because the microstructural features directly influence mechanical properties of the produced parts. An improved understanding of dendrite formation may inform design protocols to achieve optimized process parameters for controlled microstructures and enhanced properties of materials. To this end, this work implements a phase-field model to simulate directional solidification of binary alloys. For applications involving strong nonequilibrium effects, a modified antitrapping current model is incorporated to help eject solute into the liquid phase based on an experimentally calibrated, velocitydependent partitioning coefficient. Investigated allow systems include $\mathrm{SCN}, \mathrm{Si}-\mathrm{As}$, and $\mathrm{Ni}-\mathrm{Nb}$. The $\mathrm{SCN}$ alloy is chosen to verify the computational method, and the other two are selected for a parametric study due to their different diffusion properties. The modified antitrapping current model is compared with the classical model in terms of predicted dendrite profiles, tip undercooling, and tip velocity. Solidification parameters-the cooling rate and the strength of anisotropy-are studied to reveal their influences on dendrite growth. Computational results demonstrate the effectiveness of the PFM and the modified antitrapping current model in simulating rapid solidification with strong nonequilibrium at the interface.

DISCLAIMER: The contents of this report are not to be used for advertising, publication, or promotional purposes. Citation of trade names does not constitute an official endorsement or approval of the use of such commercial products. All product names and trademarks cited are the property of their respective owners. The findings of this report are not to be construed as an official Department of the Army position unless so designated by other authorized documents. 


\section{Contents}

Abstract.................................................................................................................. if

Contents........................................................................................................................... ifi

Figures and Table....................................................................................................................... iv

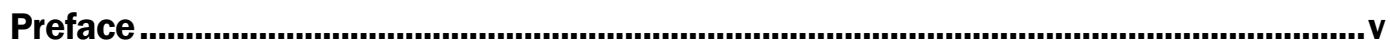

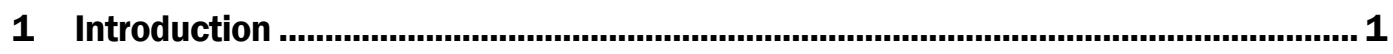

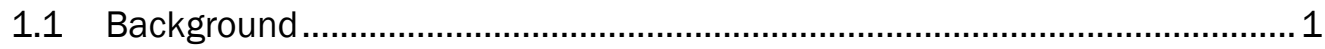

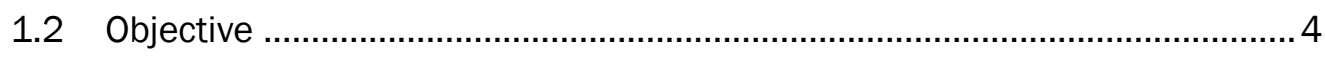

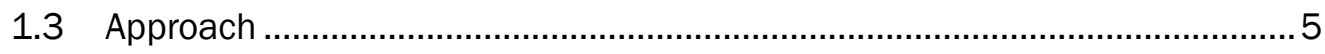

2 Solidification Modeling with the Phase-Field Method .............................................6

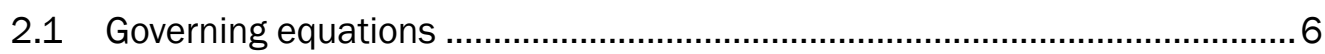

2.1.1 Derivation and mathematical details.................................................................. 6

2.1.2 Final nondimensional equations and modified antitrapping model ...................... 10

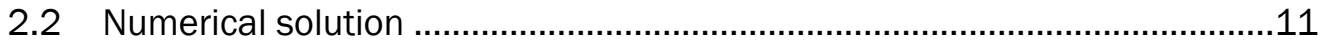

3 Material Properties...........................................................................................14

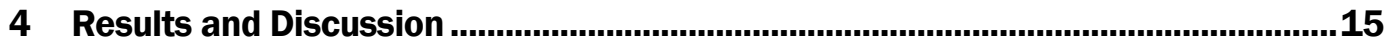

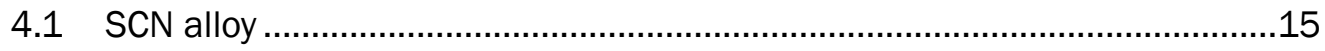

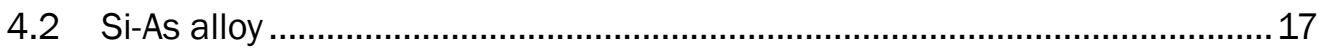

4.2.1 Effects of solidification rates....................................................................... 17

4.2.2 Effects of antitrapping models ....................................................................... 18

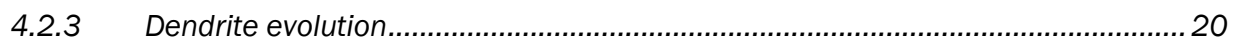

4.2.4 Effects of surface energy anisotropy ................................................................. 21

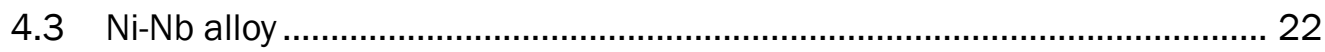

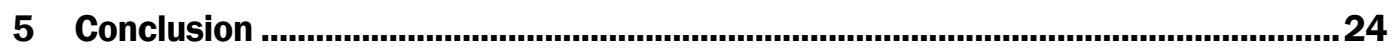

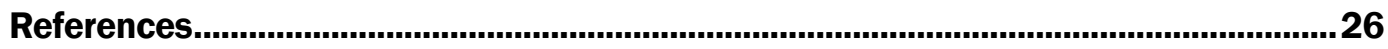

\section{Report Documentation Page}




\section{Figures and Table}

\section{Figures}

Figure 1. Demonstration of a directional solidification example.

Figure 2. Dendrite profiles for three different pulling velocities: (a) $V_{p}=29 \mu \mathrm{m} \mathrm{s}^{-1}$;

(b) $V_{p}=32 \mu \mathrm{m} \mathrm{s}^{-1}$; and (c) $V_{p}=128 \mu \mathrm{m} \mathrm{s}^{-1}$. From left to right, as $V_{p}$

increases, the SDAS decreases and the tip radius decreases.

Figure 3. Evolution of the tip velocity in the case of $V_{p}=32 \mu \mathrm{m} \mathrm{s}^{-1}$. Inset shows the curve in the range of 300 310 s.

Figure 4. Comparison of dendrite profiles under two different solidification conditions. For problem 1, (a) experimental (Pocheau and Georgelin 2001; Georgelin and Pocheau 2004) and (b) our simulation results are compared. For problem 2, (c) simulation from the literature (Echebarría, Karma, and Gurevich 2010) and (d) our simulation are compared.

Figure 5. Dendrite profiles under different solidification conditions. The first row $(a-c)$ shows dendrite profiles for slow, quasi-rapid, and rapid solidification, respectively, with the modified antitrapping current. The second row $(d-f)$ is for slow, quasi-rapid, and rapid solidification, respectively, with the classical antitrapping current.

Figure 6. (a) Difference in tip undercooling and $(b)$ velocity ratio predicted by using the classical and modified antitrapping current models for different solidification regimes. The time scale is in $\tau_{0}$.

Figure 7. Dendrite arm development with time for (a) rapid solidification and (b) quasi-rapid solidification. Note that the time scale, $\tau_{0}$, is different in the two problems. Inset shows the formation process of a new secondary arm.

Figure 8. Dendrite profiles with different strengths of anisotropy: (a) $\varepsilon_{4}=0.03$, and $(b) \varepsilon_{4}=0.01$

Figure 9. Dendrite profiles of $\mathrm{Ni}-\mathrm{Nb}$ in slow solidification predicted with the (a) modified and $(b)$ classical antitrapping current models.

Figure 10. (a) Difference in tip undercooling and $(b)$ velocity ratio predicted by using the classical and modified antitrapping current models for different solidification regimes.

\section{Table}

Table 1. Material properties of three binary alloys (SCN, Si-As, and Ni-Nb). 


\section{Preface}

This study was conducted for the Military Engineering Business Area under Project Task Order W912HZ-20-F-0058, "Phase-Field Modeling of Nonequilibrium Solidification Processes in Additive Manufacturing." The technical monitor was Dr. Robert D. Moser.

The Concrete and Materials Branch (GMC) of the Engineering Systems and Materials Division (GM), U.S. Army Engineer Research and Development Center, Geotechnical and Structures Laboratory (ERDCGSL). At the time of publication, Mr. Christopher M. Moore was Chief, GMC; Mr. Justin S. Strickler was Division Chief, GM; and Ms. Pamela G. Kinnebrew, GZT, was the Technical Director for Military Engineering. The Deputy Director of ERDC-GSL was Mr. Charles W. Ertle II, and the Director was Mr. Bartley P. Durst.

The Computational Analysis Branch (CAB) of the Computational Science and Engineering Division (CSED), U.S. Army Engineer Research and Development Center, Information Technology Laboratory (ERDC-ITL). At the time of publication, Mr. Josh Church was Acting Chief, CEERD-IE-C; Dr. Jeffrey L. Hensley was Chief, CEERD-IES; and Dr. Robert M. Wallace was the Technical Director for Engineered Resilient Systems. The Deputy Director of ERDC-ITL was Dr. Jackie Pettway, and the Director was Dr. David A. Horner.

COL Teresa A. Schlosser was the Commander of ERDC, and Dr. David W. Pittman was the Director. 


\section{Introduction}

\subsection{Background}

A dendrite is a treelike pattern spontaneously formed in nature and a ubiquitous feature of solidification. The mechanical properties of alloys depend on the complexity of dendritic microstructures formed during welding, soldering, and casting of components. As a result, researchers have sought to understand the formation of dendrite microstructures for many years. Importantly, dendritic microstructural growth exhibits different characteristics under slow and rapid solidification conditions. In recent years, rapid solidification has become increasingly important as it is common in several advanced manufacturing processes including metal additive manufacturing, laser welding, thermal spraying, and spin coating. These advanced manufacturing processes are of great interest because they are capable of fabricating complex geometries with optimum materials usage and low tooling costs in reduced time.

Several computational methods have been developed to study the physics underlying dendritic growth during solidification. Among them, the cellular automaton (CA) method and the phase-field method PFM are the two that have been used the most extensively. The CA method features relatively low computational costs, and it can be used to simulate microstructural evolution in large systems (Natsume and Ohsasa 2014). By comparison, PFM is computationally more expensive. However, it can uniquely capture more physical phenomena occurring during the solidification processes (Boettinger et al. 2002; Loginova, Amberg, and Ågren 2001; Ramirez et al. 2004; Echebarría et al. 2004). This advantage of PFM is caused by its natural connection with nonequilibrium thermodynamics and its use of order parameters, which enables the diffusion of phase boundaries and eliminates tedious front tracking. As a result, PFM has become a popular tool to simulate microstructural evolution in many material systems.

A key to the success of PFM is interface modeling. Numerically, when applying PFM, arbitrarily large interface thickness, $W$, can be chosen relative to the physical interface thickness and the characteristic capillary length. Physically, dendrite formation is controlled by nonequilibrium effects at the interface. For a microscopic $W$ and low solidification 
velocities, the solid-liquid interface rapidly reaches local thermodynamic equilibrium. However, the use of smaller $W$ requires much more time for the simulation to complete. Alternatively, mesoscopic $W$ can be used for faster computation. However, when a mesoscopic $W$ is used, the nonequilibrium effects are artificially magnified, driving more solute to concentrate at the interface. The artifacts dramatically alter microstructural evolution. To rectify the artifacts, Karma (2001) proposed an antitrapping current to counterbalance the artificially large solute trapping that results when a mesoscopic $W$ is chosen in simulating slow solidification of dilute binary alloys.

The interface modeling with PFM is even more complicated in the rapid solidification regime, where the solid-liquid interface advances at very fast rates. Due to the rapidly evolving interfaces, there is substantial aberration from the equilibrium concentrations at the interface for both the solid and liquid phases. As diffusion in practice is not as fast to allow readjustment of concentrations to maintain equilibrium, the assumption of local equilibrium usually breaks down in rapid solidification.

Solidification under such nonequilibrium conditions may result in unique microstructural features and kinetic effects. In nonequilibrium solidification, since the diffusion rate of solute is much faster in the liquid phase than in the solid phase, it is easier for the liquid phase to reach equilibrium than the solid phase. Hence, in the phase diagram, it is safe to assume that the liquidus line stays fixed but the solidus line changes. As a result, at the interface, the ratio of the solid concentration, $c_{s}$, to the liquid concentration, $c_{l}$, may vary the solidification rate. This variation can be characterized by a partitioning coefficient, which is defined by $K=c_{S} / c_{l}$. The partitioning coefficient determines the extent to which solute is ejected into the liquid during solidification.

When rapid solidification causes large interface velocities, $V$, the partitioning coefficient, $K$, increases, and more solute is ejected into the interface, leading to solute trapping. A complete understanding of the trapping process requires the definition of a velocity-dependent $K_{v}$, along with kinetic and thermodynamic properties of the alloy. The solute trapping inevitably affects solidification in terms of the microstructural morphology, length scale, microsegregation, and the resulting precipitation of secondary phases. Understanding the effects of antitrapping on solidification is very important because the resulting 
microstructural features determine, to a large extent, the behavior of the solidified materials. Moreover, these features can be related back to controllable manufacturing process parameters for the purpose of process optimization via computer modeling.

There are three main theories of solute trapping. The most widely known is the continuous growth model (CGM) developed by Aziz (1994). The CGM introduces a so-called diffusive speed, $V_{d}$, which is the velocity at which a solute atom can traverse the solid-liquid boundary. Aziz (1994) defined this parameter as $V_{d}=D / \lambda$, where $D$ is the diffusion coefficient in the interface region and $\lambda$ is the physical width of the interface. According to the CGM, significant trapping occurs when $V$ becomes comparable to $V_{d}$ and the model predicts $K_{v}$ in the limit of dilute concentrations as

$$
K_{v}=\frac{K_{e}+\frac{V}{V_{d}}}{1+\frac{V}{V_{d}}},
$$

where $K_{e}$ is the equilibrium partitioning coefficient. The extreme condition of complete trapping is given by $K_{v}=1$ as $V=\infty$.

The work of Jackson (1984) demonstrates similar asymptotic behavior, except that $K_{v}$ is described by a power law as

$$
K_{v}=K_{e}^{\frac{1}{1+A V}},
$$

where $A$ is a parameter analogous to the reciprocal of $V_{d}$.

By comparison, the work of Sobolev $(1997,1995)$ predicts that there is an abrupt change in the partitioning coefficient at finite velocities, which is known as the local nonequilibrium model (LNM). Compared with the CGM, the LNM incorporates inertial and diffusive dynamics of solute atoms near and through a rapidly advancing interface. The LNM has the following formulation:

$$
\begin{gathered}
K_{v}=\frac{K_{e}\left[1-\left(\frac{V}{V_{d}^{B}}\right)^{2}\right]+\frac{V}{V_{d}}}{1-\left(\frac{V}{V_{d}^{B}}\right)^{2}+\frac{V}{V_{d}}}, \text { for } V<V_{d}, \\
K_{v}=1, \text { for } V \geq V_{d},
\end{gathered}
$$


where $V_{d}^{B}$ is the bulk liquid diffusive speed, that is, how fast a solute atom can travel in the liquid. Complete trapping, that is, $K_{v}=1$, occurs at a well-defined velocity, $V=V_{d}$. Sobolev emphasized that solute atoms have a finite velocity in the liquid quantified by $V_{d}^{B}$, which differs from Aziz's model where $V_{d}^{B}$ in the bulk liquid is assumed infinite. Despite the differences, these models give similar results and are consistent with experiments.

Most phase-field models assume a constant $K$ that is independent of $V$, which is reasonable for slow solidification but will artificially magnify solute trapping in rapid solidification. For example, using the parabolic form of dynamic equations, the phase-field model proposed by Echebarría Domínguez, Karma, and Gurevich (2010) does not show complete trapping in the high velocity limit or rapid solidification. At high pulling velocities, the assumption of local equilibrium breaks down because the pulling velocity is so high that the physical interface velocity is unable to keep up with the pulling velocity. Hence, to use a phase-field model for rapid solidification, the antitrapping current needs modifications to account for local nonequilibrium at the interface. To this end, Pinomaa and Provatas (2019) extended the CGM model to make it applicable for PFM, where $K_{v}$ is defined as

$$
K_{v}=K_{e} e^{\left.\left(\sqrt{2}\left(1-K_{v}\right) \frac{V}{V_{d}}\right)\right)} .
$$

Compared with the original CGM, the major differences here are the definition of $V_{d}$ and $V$, which now are both based on the numerical width of the interface.

\subsection{Objective}

This work aims to model solidification of dilute binary alloys Ni-Nb, Si-As, and SCN (succinonitrile alloy) using PFM. Among the model alloys, SCN is adopted for verification against experimental studies. Dendrite evolution during directional solidification is simulated based on the model developed by Echebarría et al. (2004). This particular formulation was chosen for its generality in modeling directional solidification, which, is usually the correct physics for rapid solidification. Similar to Echebarría's study, this work assumes dilute solution, frozen temperature approximation, constant material properties, and diffusion-driven mass transport. 
Importantly, the Echebarría formulation itself only applies for slow solidification. To expand its applicability to rapid solidification, this work incorporates the antitrapping current proposed by Pinomaa and Provatas (2019), which accounts for the nonequilibrium conditions that dominate the solid-liquid interface. Computational results of dendrites are quantified by several parameters including the secondary arm spacing (SDAS). SDAS is particularly important as it governs the microsegregation pattern, which determines the strength of materials made by additive manufacturing.

\subsection{Approach}

This work commences with a statement of the free energy functional and corresponding free energy density as prescribed by Echebarría et al. (2004). The governing evolution equations relevant to phase and composition are then presented, with anisotropy included as part of the interfacial gradient energy. These are supplemented by the antitrapping current proposed by Pinomaa and Provatas (2019), as well as various other interpolation functions and phase-field parameters derived from the thin interface limit (Kim, Kim, and Suzuki 1998). The equations are then nondimensionalized and solved using the Multiphysics Object Oriented Simulation Environment (MOOSE) (Tonks et al. 2012). For objective purposes, the directional solidification of three dilute, binary alloys are considered (Ni$\mathrm{Nb}, \mathrm{Si}-\mathrm{As}$, and $\mathrm{SCN}$ ) and used to evaluate the influence of the imposed modified antitrapping current as compared with classical implementations. 


\section{Solidification Modeling with the Phase- Field Method}

\subsection{Governing equations}

The governing equations of the phase-field model include the formulation relating to two independent fields: (1) the solute concentration field, $c$, in the binary alloy and (2) the phase field, $\phi$. Here, $\phi$ is a continuous scalar field that is defined such that it distinguishes the solid phase with $\phi=+1$ and the liquid phase with $\phi=-1$. The interface has $-1<\phi<1$, leading to a thin layer of finite width that features continuous transition as opposed to the discontinuous transition assumed in some other models.

\subsubsection{Derivation and mathematical details}

The free energy functional for a two-phase system is usually described by

$$
F(\phi, c, T)=\int_{d V}\left[\frac{\sigma}{2}|\nabla \phi|^{2}+f\left(\phi, T_{m}\right)+f_{A B}(\phi, c, T)\right] .
$$

Here, $T_{m}$ is the melting temperature of pure metal; $f\left(\phi, T_{m}\right)=H\left(-\frac{\phi^{2}}{2}+\right.$ $\left.\frac{\phi^{4}}{4}\right)$ is a double-well potential with a barrier height of $H\left(T_{m}\right)$ that has the unit of energy per unit volume. Depending on the $T-c$ (i.e., temperaturesolute concentration) phase diagram, $f_{A B}(\phi, c, T)$ changes relative stability of the solid-liquid interface. The term $\sigma$ is a penalty term applied to phase gradients to ensure a finite interface thickness.

The rate equations for all fields including $c$ and $\phi$ can be derived as

$$
\begin{gathered}
\frac{\partial \phi}{\partial t}=\frac{K_{\phi} \delta F}{\delta \phi}, \\
\frac{\partial c}{\partial t}=\vec{\nabla}\left[M(\phi, c) \vec{\nabla} \frac{\delta F}{\delta c}\right],
\end{gathered}
$$

where $K_{\phi}(T)$ is a temperature-dependent kinetic constant, $\frac{\delta F}{\delta c} \equiv \mu$ is the chemical potential, and $M(\phi, c)$ is the mobility of solute atoms or molecules, which can be described as follows:

$$
M(\phi, c)=\frac{v_{0}}{R T_{m}} D \tilde{q}(\phi) c
$$


Here, $v_{0}$ is the molar volume, $R$ is the gas constant, $D$ is diffusivity of solute in the liquid, and $\tilde{q}(\phi)$ is a dimensionless interpolation function in the range of $o$ (solid) $\sim 1$ (liquid), which dictates variation of solute diffusivity at the interface. $\tilde{q}(\phi)$ usually takes the form of $\tilde{q}(\phi)=(1-$ $\phi) /[1+k-(1-k) \phi]$, where $k$ is the partitioning coefficient that describes the amount of solute ejected at the interface from the solid phase to the liquid phase. Equation (7) describes mass conservation and can be rewritten as

$$
\frac{\partial c}{\partial t}+\vec{\nabla} \cdot \overrightarrow{J_{c}}=0
$$

where $\overrightarrow{J_{c}}=M \vec{\nabla} \mu$ is the solute current density.

Importantly, $f_{A B}(\phi, c, T)$ is an interpolation function for the bulk free energy considering the entropy $[s(\phi)]$, the internal energy $[\epsilon(\phi)]$, the free energy of pure metal in the solid phase $\left[f_{A}\left(T_{m}\right)\right]$, and the contribution from solute addition in the form of

$$
f_{A B}(\phi, c, T)=f_{A}\left(T_{m}\right)-\left(T-T_{m}\right) s(\phi)+\frac{R T_{m}}{v_{0}}(c \ln (c)-c)+\epsilon(\phi) c,
$$

where

$$
\begin{gathered}
\epsilon(\phi)=\bar{\epsilon}+\frac{\bar{g}(\phi) \nabla \epsilon}{2}, \\
s(\phi)=\frac{\left(s_{s}+s_{l}\right)}{2}-\frac{\tilde{g}(\phi) L}{2 T_{m}} .
\end{gathered}
$$

Here, the subscripts $l$ and $s$ indicate liquid and solid, respectively, $\epsilon_{s}$ is the internal energy per unit volume of the solid phase, and $\epsilon_{l}$ is the internal energy per unit volume of the liquid phase. $\bar{\epsilon}=\left(\epsilon_{s}+\epsilon_{l}\right) / 2$, and latent heat per unit volume $L=T_{m}\left(s_{l}-s_{s}\right) \cdot \tilde{g}(\phi)$ and $\bar{g}(\phi)$ are interpolation functions in the form of

$$
\begin{gathered}
\tilde{g}(\phi)=\frac{1+k-2 \exp \left\{\frac{\ln (k)}{2}[1+\bar{g}(\phi)]\right\}}{1-k}, \\
\bar{g}(\phi)=\frac{2}{\ln (k)} \ln \left[\frac{1+k-(1-k) \tilde{g}(\phi)}{2}\right]-1 .
\end{gathered}
$$

Both have the properties of $\bar{g}( \pm 1)=\tilde{g}( \pm 1)= \pm 1$. 
The phase diagram is determined by the standard common tangent construction. The corresponding equilibrium solute concentrations in the solid and liquid, $c_{s}$ and $c_{l}$, are given by

$$
\left.\frac{\partial f_{s}(c, T)}{\partial c}\right|_{c=c_{S}}=\left.\frac{\partial f_{l}(c, T)}{\partial c}\right|_{c=c_{l}}=\mu_{E}(T)
$$

where $\mu_{E}$ is the chemical potential at equilibrium, and $f_{s}$ and $f_{l}$ are free energies of the solid and liquid phases, respectively. Hence, the equilibrium concentration as a function of the $x$ coordinate, $c_{0}(x)$, is expressed as

$$
c_{0}(x)=c_{l} \exp \left\{\frac{\ln (k)}{2}\left[1+\bar{g}\left(\phi_{0}(x)\right)\right]\right\},
$$

where $\phi_{0}$ is the value of $\phi$ at the interface. Equilibrium of $\phi$ requires

$$
\sigma \frac{d^{2} \phi_{0}}{d x^{2}}+H\left(\phi_{0}-\phi_{0}^{3}\right)=-\frac{R T_{m}\left(T-T_{m}\right)}{2 v_{0} m}\left[(1-k) \tilde{g}^{\prime}\left(\phi_{0}\right)+\ln (k) \frac{c_{0}(x)}{c_{l}} \bar{g}^{\prime}\left(\phi_{0}\right)\right],
$$

where $m$ is the liquidous slope $(m<0)$, and $\tilde{g}^{\prime}$ and $\bar{g}^{\prime}$ are derivatives of the functions of $\tilde{g}$ and $\bar{g}$ with respect to $\phi$.

The equations established under the equilibrium conditions can now be used to obtain particularly simple forms of the phase-field equation:

$$
\tau \frac{\partial \phi}{\partial t}=W^{2} \nabla^{2} \phi+\phi-\phi^{3}+\frac{R T_{m}\left(T-T_{m}\right)}{2 v_{0} H m} \bar{g}^{\prime}(\phi) \ln (k)\left[\frac{c-c_{0}(\phi, T)}{c_{l}(T)}\right],
$$

where $\tau=1 / K_{\phi}(T) H$ is the relaxation time and $W$ is the width of the diffuse interface.

Define $u$ as a dimensionless number in the form of

$$
u=\frac{v_{0}}{R T_{m}}\left(\mu-\mu_{E}\right)=\ln \left\{\frac{2 c}{c_{l}^{0}[1+k-(1-k) \tilde{g}(\phi)]}\right\}
$$

where $c_{l}^{0}$ is the equilibrium concentration of solute in the liquid phase. Rewrite the phase-field equation in terms of the dimensionless variable: 


$$
\tau \frac{\partial \phi}{\partial t}=W^{2} \nabla^{2} \phi+\phi-\phi^{3}-\frac{\tilde{\lambda}}{1-k} \tilde{g}^{\prime}(\phi)\left(e^{u}-1-\frac{T-T_{0}}{m c_{l}^{0}}\right)
$$

where $T_{0}$ is a reference temperature of isothermal solidification (or equilibrium liquidus temperature), $\tilde{\lambda}$ is a dimensionless ratio defined by $\tilde{\lambda}=I \Delta T_{0} W /(2 \Gamma)$ where $I=2 \sqrt{2} / 3$, and $\Gamma$ is the Gibbs-Thomson constant.

To eliminate artifacts induced by the finite interface thickness, an antitrapping current, $\vec{J}_{a t}$, is introduced to maintain equilibrium at the interface (Karma 2001). Its purpose is to transport solute atoms from the solid to the liquid:

$$
\vec{J}_{a t}=-a(\phi) W(1-k) c_{l}^{0} e^{u} \frac{\partial \phi}{\partial t} \frac{\vec{\nabla} \phi}{|\vec{\nabla} \phi|}
$$

With the antitrapping current, the equation of solute concentration is modified as

$$
\frac{\partial c}{\partial t}=\vec{\nabla} \cdot\left[D \tilde{q}(\phi) c \vec{\nabla} u-\vec{J}_{a t}\right]
$$

To make the equations nondimensional, a dimensionless supersaturation, $U$, is introduced as

$$
U=\frac{e^{u}-1}{1-k}
$$

Now consider a simplified temperature field described by the frozen temperature approximation, that is, $T(y)=T_{0}+G\left(z-V_{p} t\right)$, where $G$ is the thermal gradient, $V_{p}$ is the pulling velocity, and $z$ is the coordinate of any point of interest along the dendrite growth direction. The temperaturedependent relaxation time becomes $\tau=\tau_{0}\left[1-\frac{(1-k)\left(y-V_{p} t\right)}{l_{t}}\right]$, where $l_{t}=$ $\frac{|m|(1-k) c_{l}^{0}}{G}$ is the thermal length and $m$ is the liquidous slope.

With the frozen temperature approximation, the field equations take the form of

$$
\tau_{0}\left[1-\frac{(1-k)\left(z-V_{p} t\right)}{l_{t}}\right] \frac{\partial \phi}{\partial t}=W^{2} \nabla^{2} \phi+\phi-\phi^{3}-\lambda g^{\prime}(\phi)\left(U+\frac{z-V_{p} t}{l_{t}}\right),
$$




$$
\begin{aligned}
\left(\frac{1-k}{2}-\frac{1-k}{2} h(\phi)\right) \frac{\partial U}{\partial t}=\vec{\nabla} \cdot\left(D q(\phi) \vec{\nabla} U+a(\phi) W[1+(1-k) U] \frac{\partial \phi}{\partial t} \frac{\vec{\nabla} \phi}{|\vec{\nabla} \phi|}\right)+ \\
{[1+(1-k) U] \frac{1}{2} \frac{\partial h(\phi)}{\partial t} }
\end{aligned}
$$

where $\lambda=\frac{15}{8} \tilde{\lambda}, h(\phi)=\phi$, and $a(\phi)=1 /(2 \sqrt{2})$. The capillary length, $d_{0}$, a material property, is taken as the unit length. The ratio of $d_{o}^{2} / D$ is taken as the unit time.

Anisotropy of surface energy is accounted for by the introduction of direction-dependent interface thickness and relaxation time, that is, $W(\theta)=W a_{s}(\theta)$ and $\tau(\theta)=\tau_{0} a_{s}^{2}(\theta)$, where $\theta$ is the angle that measures the direction and $a_{s}(\theta)$ is a direction-dependent function. This study assumes the four-fold anisotropy in the form of $a_{s}=1+\epsilon_{4}(1-\cos (4 \theta))$, where $\epsilon_{4}$ is the strength of anisotropy.

\subsubsection{Final nondimensional equations and modified antitrapping model}

Following the classical perturbation scheme developed by Langer (1986) and Caginalp and Xie (1993), a ratio is defined between the interface thickness and the capillary length, that is, $\bar{e}=W / d_{0}$. Both $W$ and $\bar{e}$ are prescribed parameters and are arbitrary to some extent. Define three dimensionless parameters based on the choices of $\bar{e}$ and $W: \tilde{V}_{p}=\frac{V_{p} \tau_{0}}{W}, \tilde{l}_{t}=$ $\frac{l_{t}}{W}$, and $\widetilde{D}=a_{1} a_{2} \bar{e}$, where $a_{1}$ and $a_{2}$ are parameters that come from a thin interface analysis that maps a numerically diffuse interface to a physical interface (Karma 2001).

Writing out explicitly all interpolation functions and taking into account the contributions made by the anisotropic $W(n)$ to the functional derivatives, the field equations are rewritten as

$$
\begin{gathered}
{\left[1-\frac{(1-k)\left(z-\widetilde{V}_{p} t\right)}{\tilde{l}_{t}}\right] a_{s}(\theta)^{2} \frac{\partial \phi}{\partial t}=\vec{\nabla} \cdot\left[a_{s}(\theta)^{2} \vec{\nabla} \phi\right]+\partial_{x}\left(|\vec{\nabla} \phi|^{2} a_{s}(\theta) \frac{\partial a_{s}(\theta)}{\partial\left(\partial_{x} \phi\right)}\right)+} \\
\partial_{z}\left(|\vec{\nabla} \phi|^{2} a_{s}(\theta) \frac{\partial a_{s}(\theta)}{\partial\left(\partial_{y} \phi\right)}\right)+\phi-\phi^{3}-\lambda\left(1-\phi^{2}\right)^{2}\left(U+\frac{z-\widetilde{V}_{p} t}{\tilde{l}_{t}}\right) \\
\left(\frac{1-k}{2}-\frac{1-k}{2} \phi\right) \frac{\partial U}{\partial t}=\vec{\nabla} \cdot\left(\widetilde{D} \frac{1-\phi}{2} \vec{\nabla} U+\frac{1}{2 \sqrt{2}}[1+(1-k) U] \frac{\partial \phi}{\partial t} \frac{\vec{\nabla} \phi}{|\vec{\nabla} \phi|}\right)+ \\
{[1+(1-k) U] \frac{1}{2} \frac{\partial \phi}{\partial t}}
\end{gathered}
$$


where $x$ and $z$ are in the unit of $W$, and $t$ is in the unit of $\tau_{0}$. To determine an appropriate value of $\bar{e}$, multiple simulations have to be performed, and the value of $\bar{e}$ that leads to stable numerical results may be selected.

To extend the model to the rapid solidification regime, a modified antitrapping current model (Pinomaa and Provatas 2019) is incorporated into the phase-field model described by equations (26) and (27). Compared with the classical antitrapping model, the most important change is that an additional trapping parameter, $A$, is introduced to the antitrapping model as

$$
j_{a t}=\frac{1}{2 \sqrt{2}}\left(1-A\left(1-\phi^{2}\right)\right)[1+(1-k) U] \frac{\partial \phi}{\partial t} \frac{\vec{\nabla} \phi}{|\vec{\nabla} \phi|} .
$$

Here, $A=D_{L} / V_{D}^{P F} W$ depends on the solute diffusion coefficient in the liquid, $D_{L}$, and the diffusion velocity of the alloy, $V_{D}^{P F}$. The parameter of $V_{D}^{P F}$ can be determined by fitting the equation with experimentally determined, velocity-dependent partitioning coefficient $K_{v}$ (Tian, MuñizLerma, and Brochu 2017). By setting $A=0$, equation (28) becomes the original antitrapping current model.

\subsection{Numerical solution}

Equations (26) and (27) are numerically solved with the finite element method (FEM) by using the weak forms of the equations. Equations (29) and (30) correspond to the $\phi$ and $U$ equations, respectively.

$$
\begin{gathered}
\left(\frac{\partial \phi}{\partial t}, \psi\right)=-L\left(\left[W(\theta)^{2} \nabla \phi\right], \nabla \psi\right)+L\left(\phi-\phi^{3}-\lambda g(\phi)\left(U+\frac{z-\widetilde{V}_{p} t}{\tilde{l}_{t}}\right), \psi\right), \\
\text { where } L=\frac{1}{1-(1-k) \frac{z-\widetilde{\tilde{V} t}}{\tilde{l}_{t}}} \\
\left(\frac{\partial U}{\partial t}, \psi\right)=M\left(\frac{\widetilde{D}(1-\phi)}{2} \nabla U, \nabla \psi\right)-M\left(\frac{1}{2 \sqrt{2}}[1+(1-k) U] \frac{\partial \phi}{\partial t} \frac{\nabla \phi}{|\nabla \phi|}, \nabla \psi\right)+ \\
M\left(\frac{1}{2}[1+(1-k) U] \frac{\partial \phi}{\partial t}, \psi\right), \text { where } M=\frac{1}{\frac{(1+k)}{2}-\frac{(1-k)}{2} \phi} .
\end{gathered}
$$

Here, $(A, B)=\int_{V}(A \cdot B) d V, \psi$ is the test function, $\nabla \psi$ is the gradient of the test function, and $W(\theta)=W a_{s}(\theta)$ is the direction-dependent interface 
thickness. There are no surface integrals in the weak forms due to the assumption of no-flux boundary conditions.

The equations are solved in a rectangle computational domain with two dimensions: (1) cell height $\left(L_{z}\right)$ along the solidification direction, $z$, and (2) cell spacing $\left(L_{x}\right)$ along the $x$ direction. The parameter of $\bar{e}$ is chosen such that $W$ is smaller than a characteristic length $l_{c} \gg d_{o}$ that depends generally on growth conditions. The computational domain is initially all fluid with $\phi=-1$ except that a small, semi-elliptical seed with $\phi=1$ is placed in the center of the bottom edge to initiate solidification. The dimensionless supersaturation is initially assumed to be constant in the entire domain, that is, $U=-1$. No-flux boundary conditions are applied on all boundaries, that is, $\frac{\partial \phi}{\partial x}=\frac{\partial U}{\partial x}=\frac{\partial \phi}{\partial z}=\frac{\partial U}{\partial z}=0$. The temperature field is defined by the frozen temperature approximation, that is, $T=T_{0}+G(z-$ $\left.V_{p} t\right)$. For all examples in this study, the misorientation angle between the thermal gradient and the pulling velocity is fixed at $0^{\circ}$ so that the dendrite will grow along the $z$ direction.

The FEM solution is performed by using MOOSE. The computational domain is meshed with an element spacing of $\Delta x=\Delta z=0.8 \mathrm{~W}$ along both directions. Time evolution is based on an explicit Euler scheme with a time step chosen below the threshold of numerical instability for the diffusion equation in both dimensions, $\Delta t<\min \left(\Delta x^{2}, \Delta z^{2}\right) / 4 D$, where $D$ is the diffusion coefficient. The solution procedure takes advantage of MOOSE's adaptive meshing capability.

To illustrate the results of a typical solidification simulation, Figure 1 plots the computational domain at the end of an example simulation. Apparently, a dendrite is formed from the bottom edge. On the two sides of the dendrite, there are several secondary arms that can be characterized by the separation parameter, $\lambda_{2}$. This report will analyze the formation of such dendrite structures as influenced by several process and material parameters. 
Figure 1. Demonstration of a directional solidification example.

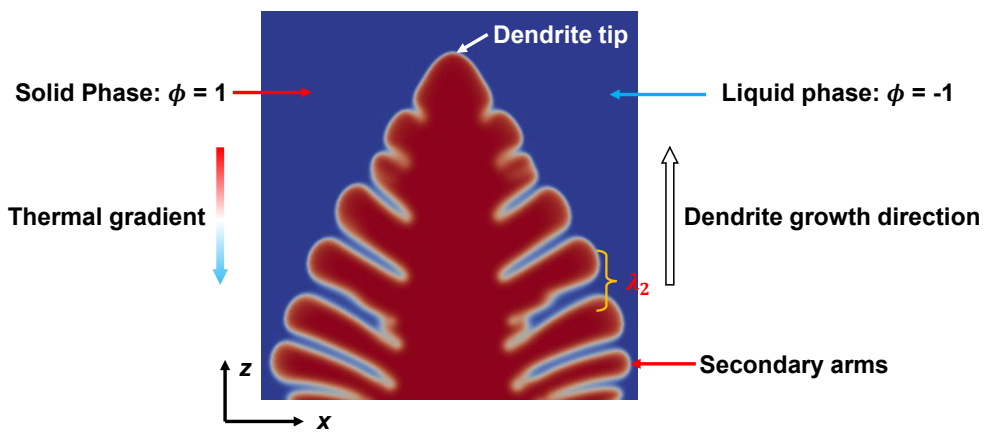




\section{Material Properties}

Material properties of three binary alloy systems studied in this work, $\mathrm{SCN}, \mathrm{Ni}-\mathrm{Nb}$, and Si-As, are given in Table 1. The thermo-physical properties of these alloys are assumed to be constant throughout the simulation. The capillary length is $d_{0}=\Gamma /(1-k)\left|m_{l}^{e}\right| c_{l}^{0}$, where $m_{l}^{e}$ is the liquidous slope at equilibrium and $c_{l}^{0}$ is the alloy concentration. The thermal length is defined as $l_{t}=\left|m_{l}^{e}\right|(1-k) c_{l}^{0} / G$, where $G$ is the thermal gradient.

Table 1. Material properties of three binary alloys (SCN, Si-As, and Ni-Nb).

\begin{tabular}{|l|c|l|c|}
\hline & $\begin{array}{l}\text { SCN } \\
\text { (Echebarría } \\
\text { et al. 2004) }\end{array}$ & $\begin{array}{l}\text { Si-As } \\
\text { (Pinomaa and } \\
\text { Provatas } \\
\text { 2019) }\end{array}$ & $\begin{array}{l}\text { Ni-Nb } \\
\text { (Ghosh et } \\
\text { al. 2017) }\end{array}$ \\
\hline Equperties & 0.03 & 0.03 & 0.48 \\
\hline Equilibrium liquidus slope, $\left|\boldsymbol{m}_{\boldsymbol{l}}^{\boldsymbol{e}}\right|\left[\frac{\boldsymbol{K}}{\boldsymbol{a t} \%}\right]$ & 2.00 & 4.00 & 10.50 \\
\hline Alloy concentration, $\boldsymbol{c}_{\boldsymbol{l}}^{\mathbf{0}}$ & - & 9.00 & 5.00 \\
\hline $\begin{array}{l}\text { Liquid diffusion coefficient, } \\
\boldsymbol{D}_{\boldsymbol{l}}\left[\mathbf{1} \boldsymbol{e}-\mathbf{9} \boldsymbol{m}^{\mathbf{2}} \boldsymbol{s}^{-\mathbf{1}}\right]\end{array}$ & 1.00 & 15.00 & 3.00 \\
\hline Capillary anisotropy strength, $\boldsymbol{\epsilon}$ & 0.007 & 0.03 & 0.03 \\
\hline Diffusive velocity (CGM), $\boldsymbol{V}_{\boldsymbol{d}}^{\boldsymbol{C G} \boldsymbol{M}}\left[\boldsymbol{m s}^{-\mathbf{1}}\right]$ & - & 0.68 & 9.00 \\
\hline Diffusive velocity (PFM) $\boldsymbol{V}_{\boldsymbol{d}}^{\boldsymbol{P F} \boldsymbol{M}}\left[\boldsymbol{m s}^{-\mathbf{1}}\right]$ & - & 0.385 & 4.00 \\
\hline
\end{tabular}




\section{Results and Discussion}

\subsection{SCN alloy}

Directional solidification of impure succinonitrile (SCN) is first studied under slow solidification conditions to verify the computational approach used in this project. The thermal gradient is fixed at a relatively low value of $G=140 \mathrm{~K} / \mathrm{cm}$, which is typical for slow solidification. The pulling velocity, $V_{p}$, is varied to study its effects on dendrite growth.

Figure 2 shows the resulting dendrite profiles for three different $V_{p}$. Apparently, as the pulling velocity and the cooling rate increase, dendrite growth is faster with sharper dendrite tip and SDAS.

Figure 2. Dendrite profiles for three different pulling velocities: (a) $V_{p}=29 \mu \mathrm{m}$ $\mathrm{s}^{-1}$; (b) $V_{p}=32 \mu \mathrm{m} \mathrm{s}^{-1}$; and (c) $V_{p}=128 \mu \mathrm{m} \mathrm{s}^{-1}$. From left to right, as $V_{p}$ increases, the SDAS decreases and the tip radius decreases.

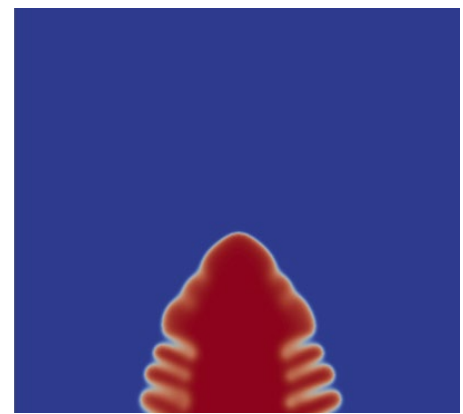

(a)

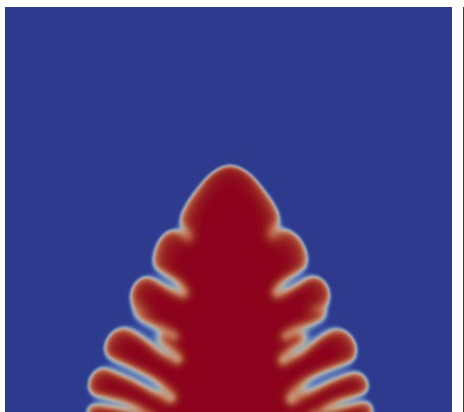

(b)

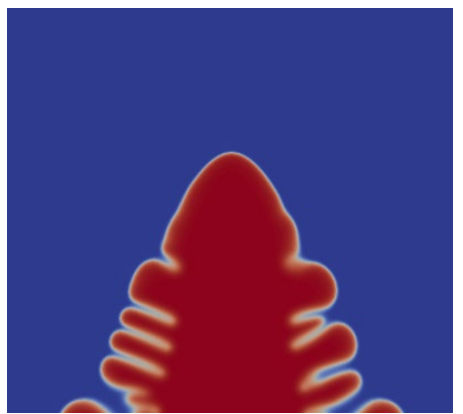

(c)

All dendrite profiles shown in Figure 2 were generated after the simulation reached the steady state, that is, when the tip velocity becomes almost a constant. As shown in Figure 3, given a constant $V_{p}$, the resulting tip velocity is not a constant. The tip velocity starts higher than $V_{p}$ at $t=0$, mainly due to the small size of the dendrite seed. Over the time, the tip velocity gradually drops to a level that is comparable to $V_{p}$ because the surface area of the interface increases so the growth is more stabilized. Similar evolution of tip velocity has been shown in several previous studies (Echebarría, Karma, and Gurevich 2010). 
Figure 3. Evolution of the tip velocity in the case of $V_{p}=32 \mu \mathrm{m} \mathrm{s}^{-1}$. Inset shows the curve in the range of 300 310 s.

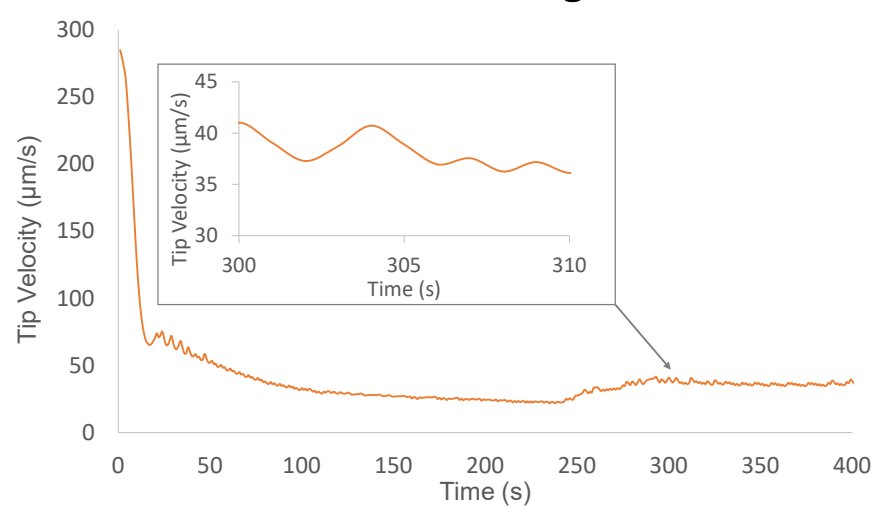

To further verify the computational approach, our simulation results are compared with experimental and computational results from the literature, in terms of the steady-state dendrite profile. On one hand, Figure $4 a-b$ compares our results with the experimental results (Pocheau and Georgelin 2001; Georgelin and Pocheau 2004) under comparable conditions in terms of the cell spacing, pulling velocity, and thermal gradient for the same alloy. On the other hand, using the same cell spacing, $L_{x}=180 \mu \mathrm{m}$, this work generates dendrite profiles that are comparable to those presented in Echebarría , Karma, and Gurevich (2010) (Figure $4 c-d$ ). The differences are mainly caused by the different simulation times. The FEM computation in this study is expensive because of the local mesh refinement at growing interfaces as well as the decrease of time step over time to ensure computational stability.

Figure 4. Comparison of dendrite profiles under two different solidification conditions. For problem 1, (a) experimental (Pocheau and Georgelin 2001; Georgelin and Pocheau 2004) and (b) our simulation results are compared. For problem 2, (c) simulation from the literature (Echebarría, Karma, and Gurevich 2010) and (d) our simulation are compared.

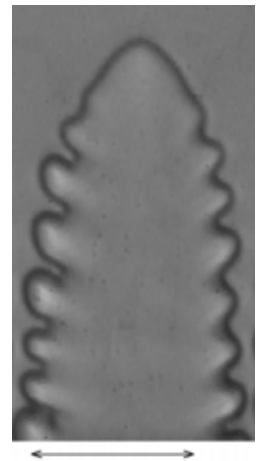

(a)

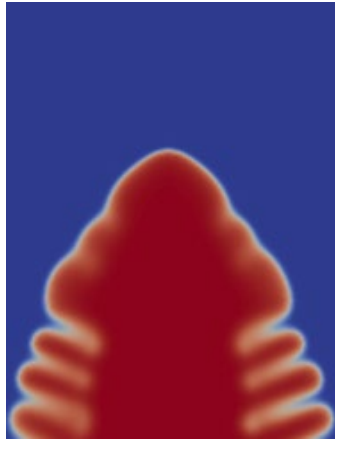

(b)

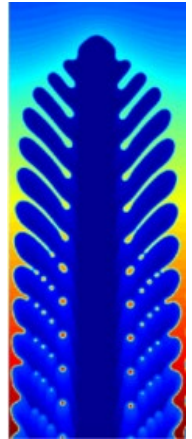

(c)

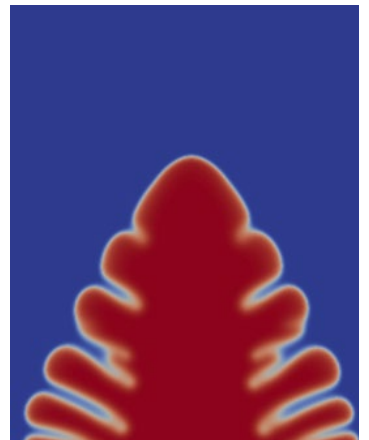

(d) 


\subsection{Si-As alloy}

After the general verification, the computational framework is further applied to study the Si-As binary alloy. Material properties are given in Table 1. The Si-As system features a very fast diffusion coefficient $\left(D_{l}\right)$ and slow diffusion velocity $\left(V_{d}\right)$, which are the major differences compared with the Ni-Nb alloy which will be studied in section 4.3. The differences in diffusion parameters will dramatically influence the interfacial local nonequilibrium in rapid solidification leading to different dendrite profiles.

Directional solidification of the Si-As alloy is studied under slow, quasirapid, and rapid solidification conditions, respectively. The simulation of slow and quasi-rapid solidification assumes a thermal gradient of $G=$ $400 \mathrm{~K} \cdot \mathrm{mm}^{-1}$ and in the case of rapid solidification, thermal gradient is fixed at $G=4,000 \mathrm{~K} \cdot \mathrm{mm}^{-1}$. The pulling velocity is prescribed as $V_{p}=$ $10 \mu \mathrm{m} \cdot \mathrm{s}^{-1}, 5,000 \mu \mathrm{m} \cdot \mathrm{s}^{-1}$, and $150,000 \mu \mathrm{m} \cdot \mathrm{s}^{-1}$ for the slow, quasi-rapid, and rapid solidification cases, respectively.

As this study is particularly focused on rapid solidification where the interface deviates from the equilibrium, we implemented the modified antitrapping current model proposed by Pinomaa and Provatas (2019). The classical antitrapping current model is also implemented as a baseline to understand the influence and applicability of the new antitrapping current model. The modified antitrapping current model has a trapping parameter of $A$ in the definition of $j_{a t}$ as described in equation (28). The value of $A$ depends on material properties including $D_{l}$ and $V_{d}$, which are distinct between the $\mathrm{Si}-\mathrm{As}$ and $\mathrm{Ni}-\mathrm{Nb}$ systems.

\subsubsection{Effects of solidification rates}

Dendrite profiles for the slow, quasi-rapid, and rapid solidification cases are shown in Figure 5, respectively. The first row uses the modified antitrapping current model, and the second row uses the classical model. Apparently, the speed of solidification significantly influences dendrite formation. Comparing dendrite profiles in any of the two rows in Figure 5, it is found that the dendrite grows much faster and wider in rapid solidification. Similar to Figure 2, Figure 5 also shows that faster solidification facilitates dendrite growth. 


\subsubsection{Effects of antitrapping models}

Moreover, by comparing Figure $5 a$ and Figure $5 d$, it is readily seen that modifying the antitrapping current has almost negligible influence in the case of slow solidification. However, as the solidification occurs at higher cooling rates, the modification of the antitrapping current model becomes more important. Comparing Figure $5 b$ and Figure $5 e$ for quasi-rapid solidification as well as Figure $5 c$ and Figure $5 f$ for rapid solidification, the modified antitrapping current model is found to boost dendrite growth and form longer dendrites.

Figure 5. Dendrite profiles under different solidification conditions. The first row $(a-c)$ shows dendrite profiles for slow, quasi-rapid, and rapid solidification, respectively, with the modified antitrapping current. The second row $(d-\hbar)$ is for slow, quasi-rapid, and rapid solidification, respectively, with the classical antitrapping current.

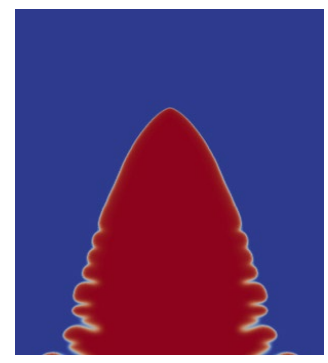

(a)

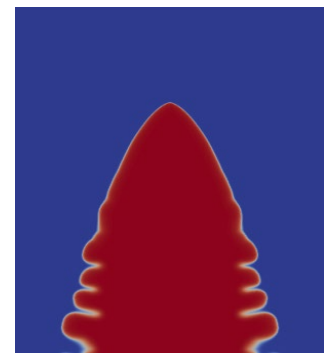

(d)

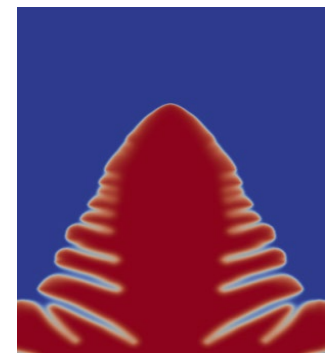

(b)

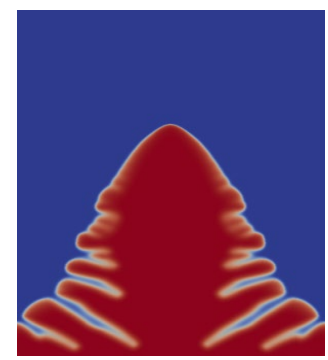

(e)

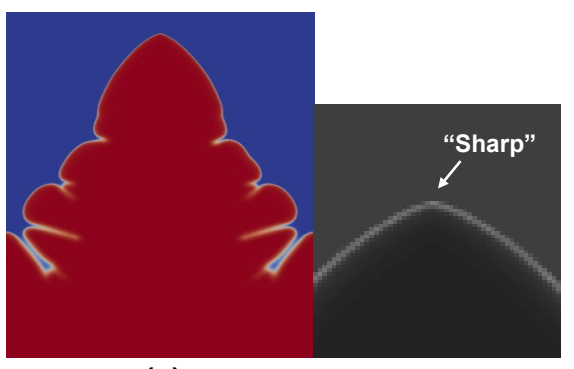

(c)

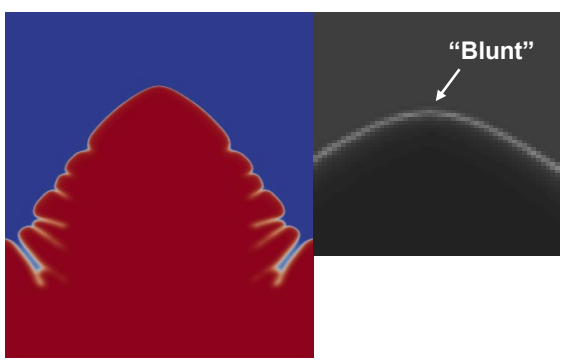

(f)

Theoretically, the "classical" antitrapping current attempts to maintain equilibrium at the interface. As a result, it predicts slower dendrite growth and more diffuse (or blunter) dendrite tip with larger tip radius as shown in the inset of Figure $5 f$. By comparison, the modified antitrapping current uses the trapping parameter to make the partitioning coefficient dependent on the diffusion velocity. As such, it ends up using a fraction of the classical antitrapping current. Therefore, the interface is further away from the equilibrium in rapid solidification, which agrees better with the physical condition. The dendrite formed with the modified antitrapping current features a sharper tip, as shown in the inset of Figure $5 c$. 
To more quantitatively understand the difference between the two antitrapping current models, Figure 6 plots the difference in tip undercooling and a tip velocity ratio. On the one hand, tip undercooling is defined by $\Omega=1-z_{\text {tip }} / l_{t}$, where $l_{t}$ is the thermal length and $z_{\text {tip }}$ is the $z$ coordinate of the dendrite tip. Figure $6 a$ plots $\Delta \Omega=\Omega^{\text {modified }}-\Omega^{\text {classical }}$, difference between the $\Omega$ predicted with the classical and the modified antitrapping current models, respectively. $\Delta \Omega$ quantifies the influence of antitrapping on the prediction of tip location. On the other hand, the tip velocity ratio is defined by $R_{V_{t}}=V_{t}^{\text {modified }} / V_{t}^{\text {classical }}$, where $V_{t}$ denotes the tip velocity. $R_{V_{t}}$ quantifies the influence of antitrapping on the prediction of tip velocity.

Figure 6. (a) Difference in tip undercooling and $(b)$ velocity ratio predicted by using the classical and modified antitrapping current models for different solidification regimes. The time scale is in $\tau_{0}$.
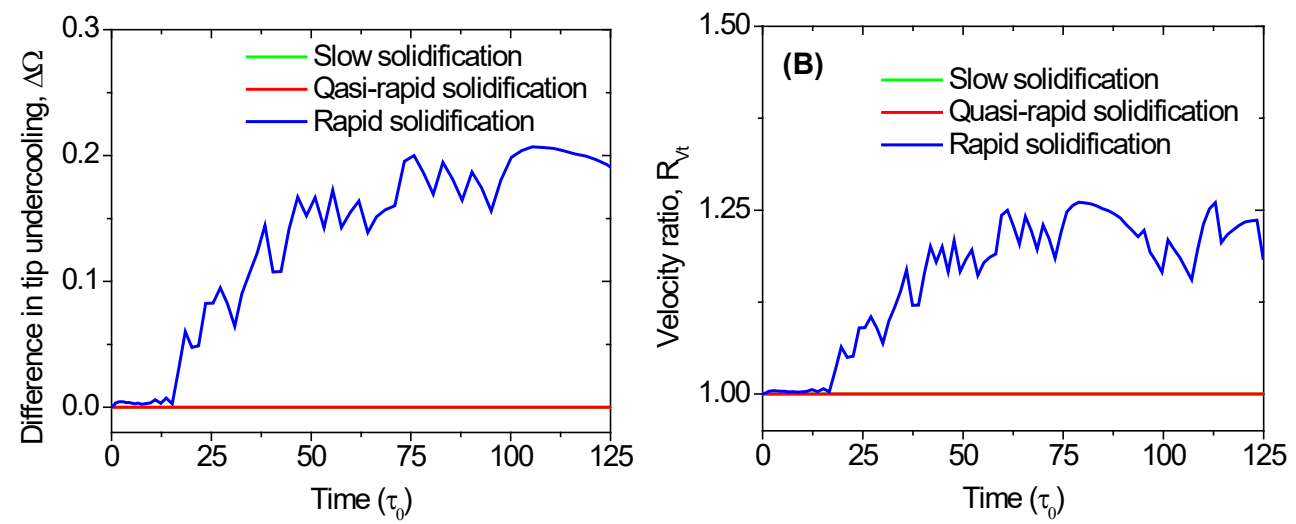

As shown in Figure 6, it is apparent that the choice of antitrapping models does not significantly influence the predictions of tip location and tip velocity in slow and quasi-rapid solidification. Both solification conditions give $\Delta \Omega \approx 0$ and $R_{V_{t}} \approx 1$, meaning that the two antitrapping models lead to almost the same simulation results.

By comparison, the choice of antitrapping models makes fundamental differences in the case of rapid solidification. Figure 6 shows that in rapid solidification, $\Delta \Omega$ stays at about $\mathrm{O}$ and $R_{V_{t}}$ stays at about 1 only at the early stage of solidification. After about $20 \tau_{0}$ for the present problem, both $\Delta \Omega$ and $R_{V_{t}}$ rise rapidly until they reach the steady state at approximately $50 \tau_{0} \sim 60 \tau_{0}$. Based on the results, using the modified antitrapping current model significantly changes the simulation results of tip advancing including its location and velocity. In terms of undercooling, the difference 
made by using the modified antitrapping model is about $18 \%$ after $50 \tau_{0}$. In terms of tip velocity, the difference is around $25 \%$ after $60 \tau_{0}$.

Theoretically, in slow and quasi-slow solidification, the interface is mostly at the equilibrium so both antitrapping models give the same results. In rapid solidification, however, the interface is no longer in equilibrium, and as a result, the classical antitrapping current, which assumes equilibrium interface, fails to predict dendrite growth accurately. At the early stage before $20 \tau_{0}$, the dendrite growth is slow so the discrepancy between the two models is negligible. As the dendrite growth rate increases, the interface becomes increasingly nonequilibrium. Hence, the classical antitrapping model's assumption of local equilibrium fails, and its prediction deviates from that made by the modified antitrapping current model.

\subsubsection{Dendrite evolution}

Figure 7 shows dendrite development snapshots for rapid and quasi-rapid solidification. Only the interface with $-1<\phi<1$ is plotted to show the dendrite morphology. In both cases, secondary arms grow from the base of the tip at the early stage of solidification. Once the shape is formed, it grows in size with time. As the dendrite grows longer, more secondary arms form, as illustrated in the inset of Figure 7. At step 1 in the inset, the tip surface of the arm is relatively flat. At step 2, the tip surface concaves approximately in the middle, which divides the arm into two. At step 3, the dividing point lags in solidification, making the two "children" arms more apparent. Overall, rapid solidification features smaller SDAS.

In all cases, the shape of the dendrite tip is parabolic, as shown in Figure 7. Depending on the growth velocity, the tip radius is sharper for rapid solidification and blunter for slow solidification. Additionally, Figure 7 also reveals that the interface is wider in between the secondary arms, suggesting higher solute concentration since solute concentration at the interface is always higher than the equilibrium concentration. Hence, the solute is enriched between neighboring secondary arms. 
Figure 7. Dendrite arm development with time for $(a)$ rapid solidification and $(b)$ quasi-rapid solidification. Note that the time scale, $\tau_{0}$, is different in the two problems. Inset shows the formation process of a new secondary arm.
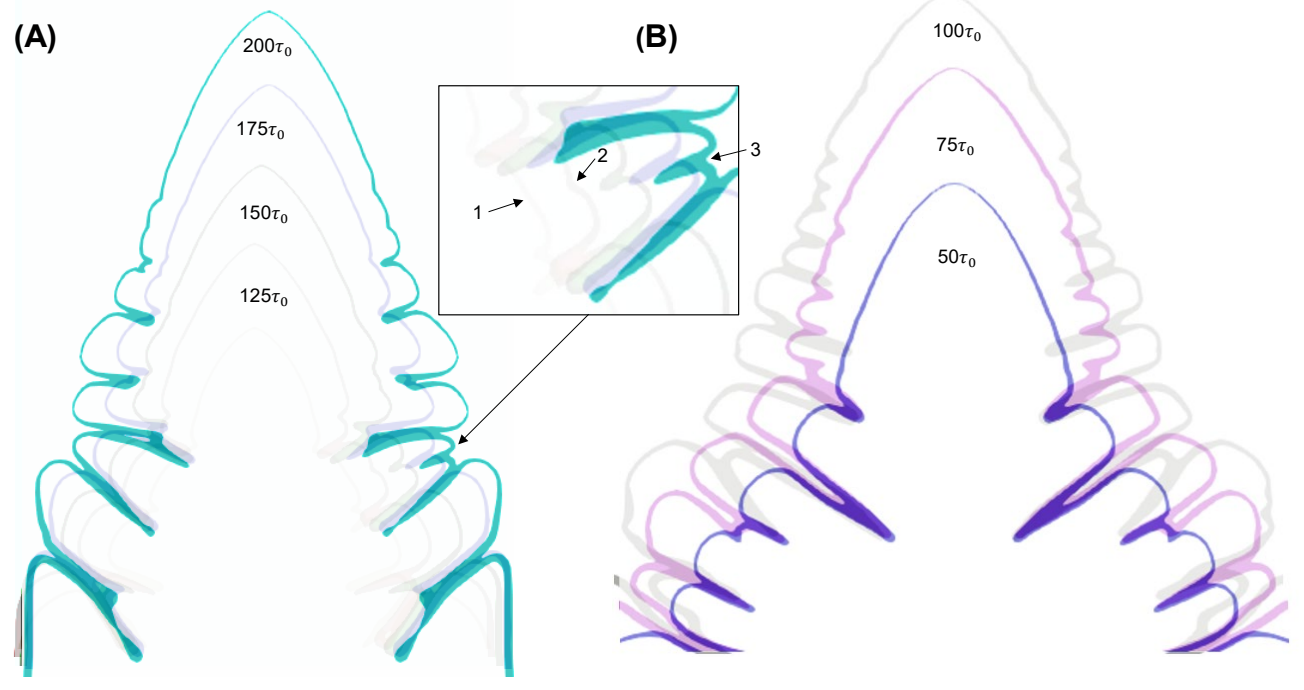

\subsubsection{Effects of surface energy anisotropy}

This study further investigates the effects of surface energy anisotropy by varying the strength of anisotropy $\left(\epsilon_{4}\right)$ between the two values of 0.03 and 0.01. Figure 8 shows dendrite profiles for the two cases. When the strength of anisotropy is reduced from $\epsilon_{4}=0.03$ to 0.01 , the number of secondary arms increases drastically. According to previous studies, the growth of secondary arms may also be boosted by artificial noises introduced at the interface to better simulate practical solidification conditions. However, since the present work does not introduce any artificial noises, the changes seen in the secondary arms are solely caused by the strength of anisotropy. Hence, based on the results shown in Figure 8, anisotropy is found to suppress the formation of secondary arms in the dendrite. Similar results have been found in other works (Xing et al. 2017).

The higher anisotropy also leads to a sharper dendrite tip. Additionally, the lower anisotropy is found to slow down tip velocity. As more secondary arms are present, the interface surface area is enlarged, leading to lower tip velocity. 
Figure 8. Dendrite profiles with different strengths of anisotropy:

(a) $\varepsilon_{4}=0.03$, and $(b) \varepsilon_{4}=0.01$.
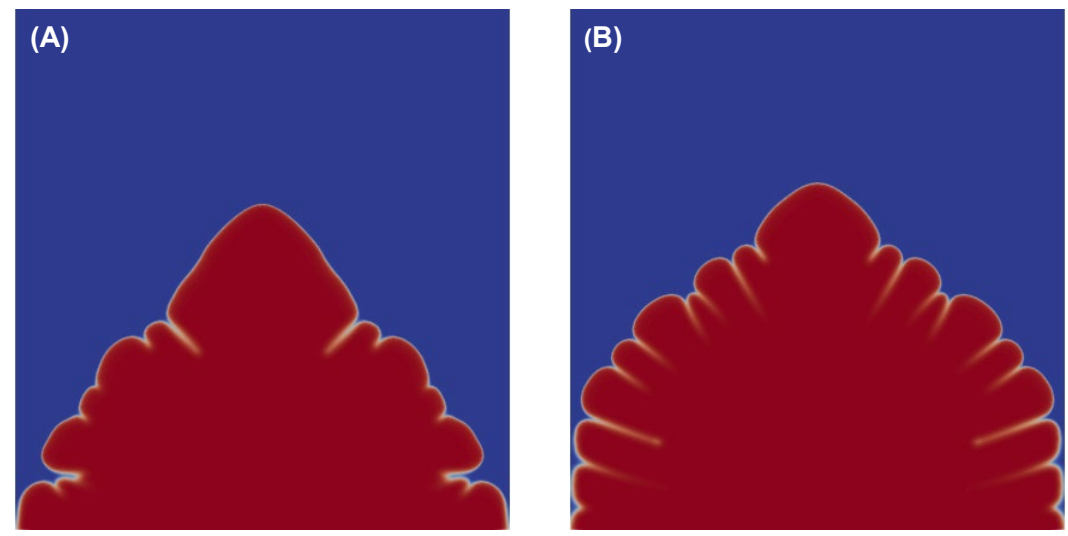

\subsection{Ni-Nb alloy}

This section studies directional solidification of the Ni-Nb binary alloy. Material properties are given in Table 1 . The $\mathrm{Ni}-\mathrm{Nb}$ system has a relatively low diffusion coefficient $\left(D_{l}\right)$ and a fast diffusion velocity $\left(V_{d}\right)$ compared to the Si-As system. With the high diffusion velocity, solute atoms have higher mobility at the interface so that they can readjust local concentration as needed to maintain local equilibrium. Hence, the $\mathrm{Ni}-\mathrm{Nb}$ system has weaker nonequilibrium effects compared with the Si-As system in rapid solidification.

Two solidification conditions were assumed in this study: (1) $G=$ $400 \mathrm{~K} \mathrm{~mm}^{-1}$ and $V_{p}=10 \mu \mathrm{m} \mathrm{s}^{-1}$ representing slow solidification and (2) $G=4,000 \mathrm{~K} \mathrm{~mm}^{-1}$ and $V_{p}=150,000 \mu \mathrm{m} \mathrm{s}^{-1}$ representing rapid solidification.

Figure 9 compares the dendrite profiles in the case of slow solidification predicted by the classical and modified antitrapping current models, and no significant difference is identified. The results are verified using the difference in tip undercooling and velocity ratio as shown in Figure 10. Both parameters remain a constant with time in the case of slow solidification, indicating that local equilibrium holds and the classical antitrapping model is sufficient for accurate prediction. 
Figure 9. Dendrite profiles of $\mathrm{Ni}-\mathrm{Nb}$ in slow solidification predicted with the (a) modified and $(b)$ classical antitrapping current models.
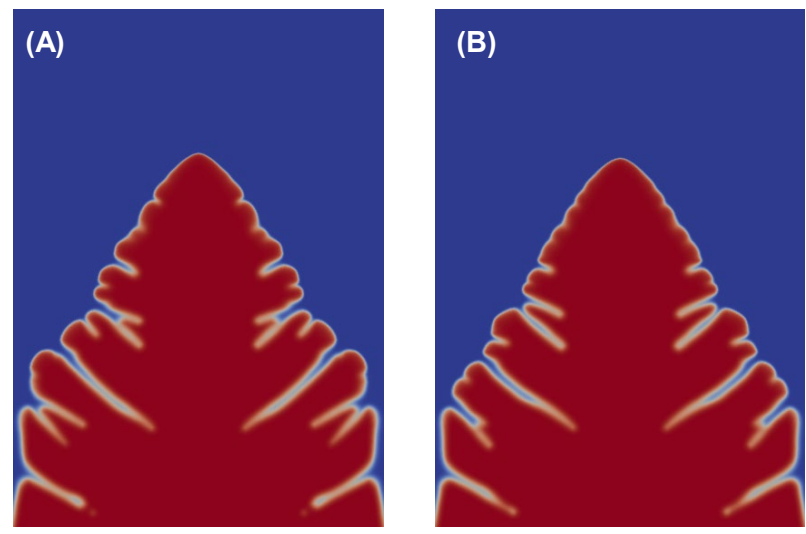

In the case of rapid solidification, Figure 10 shows that both parameters of the difference in tip undercooling and velocity ratio have much lower magnitudes (3\%-10\%) than their values in Figure 6 for Si-As (18\%-25\%). Indeed, as pointed out above, the $\mathrm{Ni}-\mathrm{Nb}$ alloy features fast solute diffusion at the interface, which alleviates interfacial nonequilibrlium in rapid solidification. The modified antitrapping model is still more accurate, but its advantage over the classical model is not as significant as that in the SiAs system.

Figure 10. (a) Difference in tip undercooling and $(b)$ velocity ratio predicted by using the classical and modified antitrapping current models for different solidification regimes.
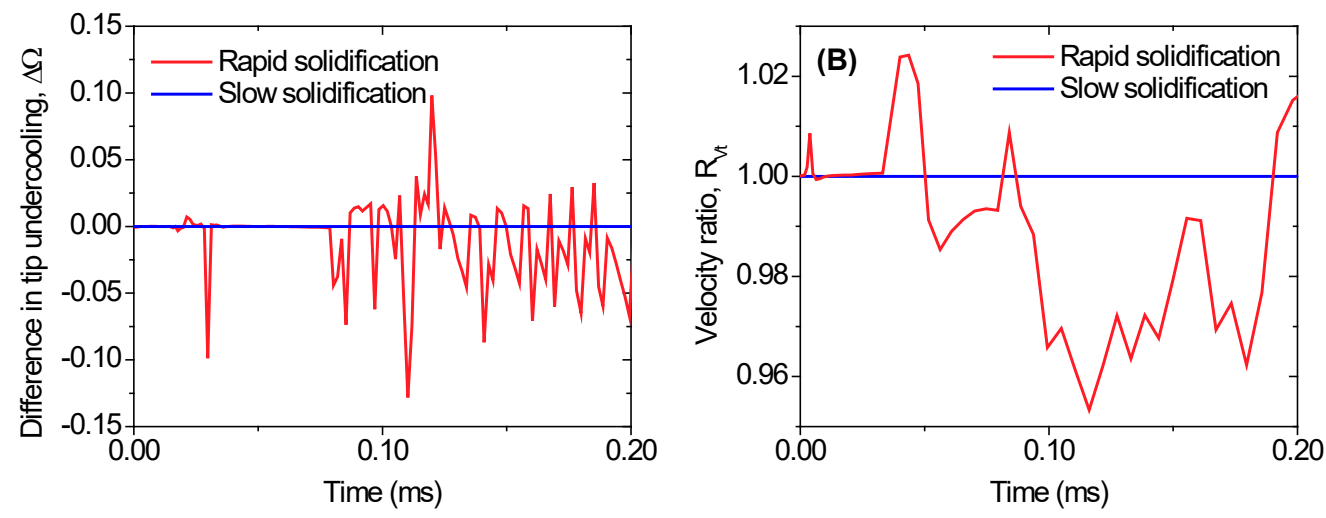


\section{Conclusion}

To summarize, this project implements the phase-field model for directional solidification of binary alloys developed by Echebarría et al. (2004) with two antitrapping models. The computational tool is verified and tested by considering the two-dimensional, one-sided directional solidification of three binary systems: SCN, Si-As, and Ni-Nb. Dendrite growth under different process parameters (i.e., cooling rate and strength of anisotropy) is studied to understand those parameters' effects. Major findings from the numerical work include:

1. Studies of the three systems (i.e., $\mathrm{SCN}, \mathrm{Si}-\mathrm{As}$, and $\mathrm{Ni}-\mathrm{Nb}$ ) all reveal parabolic dendrite tip evolving along the directions of maximum interface energy. Solute enrichment between neighboring secondary arms is observed.

2. The classical antitrapping current model fails in rapid solidification, especially for alloys that have low diffusion velocities at the interface. The classical antitrapping current model builds upon the assumption of local equilibrium, which breaks down in the case of rapid solidification. Using the modified antitrapping current model is suggested for alloys with low to moderate diffusion velocities. The modified antitrapping current uses a fraction of the classical antitrapping current through a trapping parameter, effectively making the partitioning coefficient velocity dependent, which can be experimentally calibrated.

3. This study uses two parameters to quantify the differences between the two antitrapping models: the difference in tip undercooling and velocity ratio. Since the modified antitrapping model was designed to capture nonequilibrium conditions at the interface, the two parameters also indicate quantitatively the effects of interfacial nonequilibrium on dendrite growth. This study reveals that in $\mathrm{Si}$-As, local equilibrium holds at the very early stage of solidification for up to $20 \tau_{0}$; after that, significant deviation from local equilibrium occurs that influences dendrite growth, and this influence is stabilized at approximately $50 \sim 60 \tau_{0}$. The Si-As is therefore an excellent example where the modified antitrapping current model is required for accurate prediction. 
4. In $\mathrm{Ni}-\mathrm{Nb}$, the influence of interfacial nonequilibrium is much less significant, and the modified antitrapping model does not generate significantly different results. With high diffusion velocities at the interface, the solute atoms in $\mathrm{Ni}-\mathrm{Nb}$ are able to quickly readjust themselves to maintain local equilibrium, resulting in similar predictions with the two antitrapping current models.

5. As the cooling rate increases, the secondary arm spacing decreases, and the dendrite tip becomes sharper.

6. The lower anisotropic strength of surface energy results in more secondary arms in the dendrite structure. It also slows down dendrite tip growth as the interface surface area is increased with more secondary arms.

In future studies, the directional solidification model may be extended to include multicomponent systems to study interesting phenomenon pertaining to additive manufacturing. For example, the Inconel 718 (IN718) alloy, composed of 50\%-55\% nickel, 17\%-21\% chromium, 4.75\%$5.5 \%$ niobium and tantalum, and trace amounts of other elements, is very common in $3 \mathrm{D}$ printing. Another example of multicomponent alloys is the high-entropy alloys. These systems have shown interesting phenomena under additive manufacturing, which may be a topic of future investigation. 


\section{References}

Aziz, M. J. 1994. "Non-Equilibrium Interface Kinetics during Rapid Solidification." Materials Science and Engineering: A 178, no. 1 (April): 167-70.

https://doi.org/10.1016/0921-5093(94)90537-1.

Boettinger, W. J., J. A. Warren, C. Beckermann, and A. Karma. 2002. "Phase-Field Simulation of Solidification." Annual Review of Materials Research 32, no. 1 (August): 163-94. https://doi.org/10.1146/annurev.matsci.32.101901.155803.

Caginalp, G., and W. Xie. 1993. "Phase-Field and Sharp-Interface Alloy Models." Physical Review E: Statistical, Nonlinear, and Soft Matter Physics 48, no. 3 (September): 1897-909. https://doi.org/10.1103/PhysRevE.48.1897.

Echebarría, B., A. Karma, and S. Gurevich. 2010. "Onset of Sidebranching in Directional Solidification.” Physical Review E: Statistical, Nonlinear, and Soft Matter Physics 81, no. 2 (February): 021608-1-17. https://doi.org/10.1103/PhysRevE.81.021608.

Echebarría, B., R. Folch, A. Karma, and M. Plapp. 2004. "Quantitative Phase-Field Model of Alloy Solidification.” Physical Review E: Statistical, Linear, and Soft Matter Physics 70, no. 6 (December): 061604. https://doi.org/10.1103/PhysRevE.70.061604.

Georgelin, M., and A. Pocheau. 2004. "Characterization of Cell Tip Curvature in Directional Solidification.” Journal of Crystal Growth 268, no. 1 (July): 272-83. https://doi.org/10.1016/j.jcrysgro.2004.04.043.

Ghosh, S., L. Ma, N. Ofori-Opoku, and J. E. Guyer. 2017. "On the Primary Spacing and Microsegregation of Cellular Dendrites in Laser Deposited Ni-Nb Alloys." Modelling and Simulation in Materials Science and Engineering 25, no. 6 (June): 065002. https://doi.org/10.1088/1361-651X/aa7369.

Jackson, K. A. 1984. "Crystal Growth Kinetics.” Materials Science and Engineering 65, no. 1 (July): 7-13. https://doi.org/10.1016/0025-5416(84)90194-0.

Karma, A. 2001. "Phase-Field Formulation for Quantitative Modeling of Alloy Solidification.” Physical Review Letters 87, no. 11 (August): 115701. https://doi.org/10.1103/PhysRevLett.87.115701.

Kim, S. G., W. T. Kim, and T. Suzuki. 1998. "Interfacial Compositions of Solid and Liquid in a Phase-Field Model with Finite Interface Thickness for Isothermal Solidification in Binary Alloys." Physical Review E: Statistical Physics, Plasmas, Fluids, and Related Interdisciplinary Topics 58: 3316-23. https://doi.org/10.1103/PhysRevE.58.3316.

Langer, J. S. 1986. "Models of Pattern Formation in First-Order Phase Transitions.” In Directions in Condensed Matter Physics 1 (August): 165-86. https://doi.org/10.1142/9789814415309_0005.

Loginova, I., G. Amberg, and J. Ågren. 2001. "Phase-Field Simulations of Non-Isothermal Binary Alloy Solidification.” Acta Materialia 49, no. 4 (February): 573-81. https://doi.org/10.1016/S1359-6454(00)00360-8. 
Natsume, Yukinobu, and Kenichi Ohsasa. 2014. "Three-Dimensional Cellular Automaton Model for the Prediction of Microsegregation in Solidification Grain Structures." ISIJ International 54 (2): 415-21. https://doi.org/10.2355/isijinternational.54.415.

Pinomaa, T., and N. Provatas. 2019. "Quantitative Phase Field Modeling of Solute Trapping and Continuous Growth Kinetics in Quasi-Rapid Solidification." Acta Materialia 168 (April): 167-77. https://doi.org/10.1016/i.actamat.2019.02.009.

Pocheau, A., and M. Georgelin. 2001. "Validation Criterion for Noise-Induced Mechanism of Sidebranching in Directional Solidification." The European Physical Journal B: Condensed Matter and Complex Systems 21, no. 2 (May): 229-40. https://doi.org/10.1007/s100510170199.

Ramirez, J. C., C. Beckermann, A. Karma, and H.-J. Diepers. 2004. "Phase-Field Modeling of Binary Alloy Solidification with Coupled Heat and Solute Diffusion." Physical Review E: Statistical, Nonlinear, and Soft Matter Physics 69, no. 5 (May): 051607. https://doi.org/10.1103/PhysRevE.69.051607.

Sobolev, S. L. 1995. "Local-Nonequilibrium Model for Rapid Solidification of Undercooled Melts." Physics Letters A 199, no. 5 (February): 383-86. https://doi.org/10.1016/0375-9601(95)00084-G.

Sobolev, S. L. 1997. "Rapid Solidification under Local Nonequilibrium Conditions." Physical Review E: Statistical, Nonlinear, and Soft Matter Physics 55, no. 6 (June): 6845-54. https://doi.org/10.1103/PhysRevE.55.6845.

Tian, Y., J. A. Muñiz-Lerma, and M. Brochu. 2017. "Nickel-Based Superalloy Microstructure Obtained by Pulsed Laser Powder Bed Fusion." Materials Characterization 131 (September): 306-15. https://doi.org/10.1016/j.matchar.2017.07.024.

Tonks, M. R., D. Gaston, P. C. Millett, D. Andrs, and P. Talbot. 2012. “An Object-Oriented Finite Element Framework for Multiphysics Phase Field Simulations." Computational Materials Science 51 (1): 20-29. https://doi.org/10.1016/i.commatsci.2011.07.028.

Xing, H., L. Zhang, K. Song, H. Chen, and K. Jin. 2017. "Effect of Interface Anisotropy on Growth Direction of Tilted Dendritic Arrays in Directional Solidification of Alloys: Insights from Phase-Field Simulations." International Journal of Heat and Mass Transfer 104: 607-14. https://doi.org/10.1016/i.ijheatmasstransfer.2016.08.096. 


\section{REPORT DOCUMENTATION PAGE}

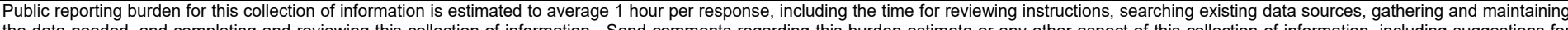

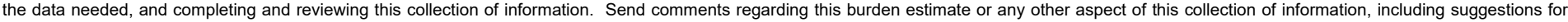

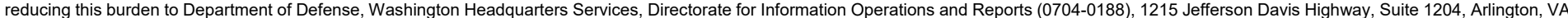

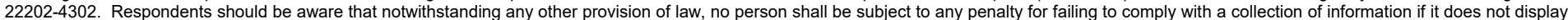
a currently valid OMB control number. PLEASE DO NOT RETURN YOUR FORM TO THE ABOVE ADDRESS.
1. REPORT DATE (DD-MM-YYYY)
November 2021

\section{REPORT TYPE}
Technical Report / Final

\section{TITLE AND SUBTITLE}

Phase-Field Modeling of Nonequilibrium Solidification Processes in Additive Manufacturing

3. DATES COVERED (From - To)

\section{5a. CONTRACT NUMBER}

5b. GRANT NUMBER

5c. PROGRAM ELEMENT NUMBER

9C2284

5d. PROJECT NUMBER

49083

Jeffrey B. Allen, Robert D. Moser, Zackery B. McClelland, Md Mohaiminul Islam, and Ling Liu

5e. TASK NUMBER

A 1020

5f. WORK UNIT NUMBER

8. PERFORMING ORGANIZATION REPORT NUMBER

ERDC-TR-21-21

Information Technology Laboratory

U.S. Army Engineer Research and Development Center

3909 Halls Ferry Road

Vicksburg, MS 39180-6199

Geotechnical and Structures Laboratory

U.S. Army Engineer Research and Development Center

3909 Halls Ferry Road

Vicksburg, MS 39180-6199

Mechanical Engineering

Temple University

1947 N 12th St

Philadelphia, PA 19122 USA

\section{SPONSORING / MONITORING AGENCY NAME(S) AND ADDRESS(ES)}

Information Technology Laboratory

U.S. Army Engineer Research and Development Center

3909 Halls Ferry Road

Vicksburg, MS 39180-6199

\section{DISTRIBUTION / AVAILABILITY STATEMENT}

Approved for public release; distribution is unlimited.

\section{SUPPLEMENTARY NOTES}

Project Task Order W912HZ-20-F-0058

\section{ABSTRACT}

This project models dendrite growth during nonequilibrium solidification of binary alloys using the phase-field method (PFM). Understanding the dendrite formation processes is important because the microstructural features directly influence mechanical properties of the produced parts. An improved understanding of dendrite formation may inform design protocols to achieve optimized process parameters for controlled microstructures and enhanced properties of materials. To this end, this work implements a phase-field model to simulate directional solidification of binary alloys. For applications involving strong nonequilibrium effects, a modified antitrapping current model is incorporated to help eject solute into the liquid phase based on experimentally calibrated, velocity-dependent partitioning coefficient. Investigated allow systems include SCN, Si-As, and Ni-Nb. The SCN alloy is chosen to verify the computational method, and the other two are selected for a parametric study due to their different diffusion properties. The modified antitrapping current model is compared with the classical model in terms of predicted dendrite profiles, tip undercooling, and tip velocity. Solidification parameters - the cooling rate and the strength of anisotropy - are studied to reveal their influences on dendrite growth. Computational results demonstrate effectiveness of the PFM and the modified antitrapping current model in simulating rapid solidification with strong nonequilibrium at the interface.

\section{SUBJECT TERMS}

Addititive manufacturing, Dendrites--Growth, Binary systems (Metallurgy), Solidification, Directional solidification

16. SECURITY CLASSIFICATION OF:

\section{a. REPORT}

Unclassified

\section{b. ABSTRACT}

Unclassified

\section{c. THIS PAGE}

Unclassified
17. LIMITATION OF ABSTRACT

None
18. NUMBER OF PAGES

35 19a. NAME OF RESPONSIBLE PERSON

19b. TELEPHONE NUMBER (include area code) 